\title{
The Stereocomplex Formation in Poly(Methyl Methacrylate) and the Stereospecific Polymerization of its Monomer
}

\author{
Takeaki MIYAmoto and Hiroshi InAGAKI \\ Institute for Chemical Research, Kyoto University, Uji, Kyoto-fu, 611, Japan.
}

(Received August 6, 1969)

\begin{abstract}
The stereocomplex formation between isotactic and syndiotactic poly(methyl methacrylate) (PMMA) molecules is studied in detail, using the techniques of thin layer chromatography (TLC), ultracentrifugation, X-ray diffraction, viscometry, and high-resolution NMR. It is shown that the 1:1 (iso/synd) complex is formed as the primary structure; further associations between the 1:1 complex and free syndiotactic molecules, when they exist in solution, occur to form the 1:2 (iso/synd) complex.

The so-called "stereoblock" PMMA samples prepared with $n$-butylmagnesium chloride and diphenylmagnesium are analyzed by using tlc technique. Evidence is thus shown for the stereochemical identity between the stereoblock PMMA and the stereocomplex which is formed by isotactic and syndiotactic PMMA in the ratio 1:2 (iso/synd). On the basis of this result, we conclude that the 1:2 stereocomplex may be formed during stereospecific polymerization of methyl methacrylate (MMA) with different Grignard's types of catalysts.

In relation to the above conclusion stereospecific polymerizations of $\mathrm{MMA}$ at $-50^{\circ} \mathrm{C}$ with $n$-butylmagnesium chloride in toluene were carried out in the presence of either isotactic or syndiotactic PMMA. The results suggest that the stereospecific polymerization of MMA is closely linked to the stereocomplex formation. The presence of the isotactic polymer chains promotes preferentially the polymerization of MMA to the syndiotactic polymer chains, while the presence of the syndiotactic polymer chains appears to be a prerequisite for initiating the polymerization to the isotactic polymer chains so far as this catalytic system is employed at $-50^{\circ} \mathrm{C}$.
\end{abstract}

\section{KEY WORDS Stereocomplex Formation / Poly(Methyl Methacrylate) / Thin Layer Chromatography / Ultracentrifugation / X-ray Diffraction / Stereoblock / Grignard / Stereospecific Polymerization /}

The first documented study of specific interactions between tactic methyl methacrylate polymer chains is probably that reported by Watanabe, et al. ${ }^{1}$ who recorded the gel formation phenomena observed upon admixture of solutions of the isotactic and the syndiotactic polymer. A fairly large number of publications on the phenomena have been presented to date and at present it is recognized that stereocomplexes are formed between tactic chains of poly(methyl methacrylate) (PMMA). $^{2-6}$ However, these works have mainly concerned the effects of solvent, polymer concentration and the stereoisomeric composition upon the stereocomplex formation, and the structure and the formation mechanism of stereocomplexes are still open for discussion to some extent.

A crystallizable type of PMMA was obtained with 9-fluorenyllithium and other organometallic catalysts under specified conditions and presumed as a "stereoblock" polymer characterized by high contents of both isotactic and syndiotactic sequences in the same chain. ${ }^{7-9}$ A possible polymerization mechanism for such a stereoblock formation has been proposed. ${ }^{10,11}$ Recently Liquori and his associates ${ }^{12}$ have, however, confirmed the stereochemical identity between the stereoblock PMMA and the stereocomplex which is formed by isotactic and syndiotactic PMMA in the ratio 1:2 (iso/synd). This result allows us to presume that under some specific conditions, stereospecific polymerizations of methyl methacrylate (MMA) may proceed through á reaction mechanism in which the stereocomplex formation plays an essential role.

One of the aims of this study is to present more 
detailed structural features of the stereocomplex formation and of the stereoblock polymer by using the techniques of thin layer chromatography (TLC), ultracentrifuge, high-resolution nuclear magnetic resonance (NMR), and X-ray diffraction. The other aim is to contribute to the elucidation of the possible mechanism for the stereospecific polymerization of MMA by referring to our knowledge gained of the stereocomplex formation. For this purpose either isotactic or syndiotactic PMMA is added to the reaction mixture in advance of starting the stereospecific polymerization of MMA, and the effect of the polymer addition upon the stereochemical structure of resultant polymers is investigated.

\section{EXPERIMENTAL}

\section{Polymer Samples}

Purified MMA was polymerized at $25^{\circ} \mathrm{C}$ with phenylmagnesium bromide in toluene according to the method given by Crescenzi, et $a l^{13}$ to obtain isotactic PMMA. The product was fractionated into seven fractions by normal fractionating precipitation at $40^{\circ} \mathrm{C}$ using acetone and methanol as solvent and precipitant, respectively. The third fraction was coded $i$ MA- 3 and used for the subsequent experiments.

A syndiotactic sample was prepared by pho- topolymerization in bulk at $-50^{\circ} \mathrm{C}$ using $\mathrm{UV}$ light in the presence of benzoin as photosensitizer. ${ }^{14}$ The whole polymer was fractionated into nine fractions by the same procedure employed for the isotactic polymer. The second fraction was coded $s \mathrm{MA}-2$ and used for our experiments. In addition, a monodisperse PMMA was synthesized at $-78^{\circ} \mathrm{C}$ with sodium biphenyl in tetrahydrofuran $^{15}$ and used as the representative of atactic PMMA, this being designated AMA.

The isotactic and syndiotactic polymers which were used as added polymer in the stereospecific polymerization in the presence of tactic polymer were prepared under the same conditions as the above but were not fractionated. Viscosityaverage molecular weights $\left(M_{v}\right)$ of these whole polymers were $8 \times 10^{5}$ and $1.4 \times 10^{5}$ for the isotactic and syndiotactic polymer coded $i \mathrm{MA}$ and $s \mathrm{MA}$, respectively. The dyad tacticity of the former was essentially $100 \%$ isotactic; the latter at least $90 \%$ syndiotactic.

Two samples of the so-called "stereoblock" PMMA were obtained by using different catalysts, $n$-butylmagnesium chloride and diphenylmagnesium. The polymerization runs were carried out at $-50^{\circ} \mathrm{C}$ in toluene as solvent. The polymerizates thus obtained were subjected to extraction with acetone at boiling point for $12 \mathrm{hr}$, according to the method reported by Liquori, et $a l .^{2 \mathrm{a}}$ Insoluble portions in boiling acetone

Table I. Characterization of sample polymers used

\begin{tabular}{|c|c|c|c|c|c|c|c|c|}
\hline \multirow{2}{*}{$\begin{array}{l}\text { Sample } \\
\text { code }\end{array}$} & \multirow{2}{*}{ Initiator } & \multirow{2}{*}{$\begin{array}{l}\text { Polymerization } \\
\text { condition }\end{array}$} & \multirow{2}{*}{$\begin{array}{c}{[\eta] 25^{\circ} \mathrm{C}} \\
\text { (dl/g), } \\
\text { in } \\
\text { chloroform }\end{array}$} & \multirow{2}{*}{$M_{v} \times 10^{-5}$} & \multirow{2}{*}{$\begin{array}{l}\text { IR index } \\
\text { (A) }\end{array}$} & \multicolumn{3}{|c|}{ Tacticity } \\
\hline & & & & & & I & $\mathrm{H}$ & $\mathbf{S}$ \\
\hline$i \mathrm{MA}^{\mathrm{a}}$ & $\mathrm{C}_{6} \mathrm{H}_{5} \mathrm{Mg} \mathrm{Br}$ & $\begin{array}{l}\text { Toluene soln. } \\
\text { at } 25^{\circ} \mathrm{C}\end{array}$ & 2.58 & 8.0 & 0 & 1.0 & 0 & 0 \\
\hline$i \mathrm{MA}-3$ & $\mathrm{C}_{6} \mathrm{H}_{5} \mathrm{Mg} \mathrm{Br}$ & $\begin{array}{l}\text { Toluene soln. } \\
\text { at } 25^{\circ} \mathrm{C}\end{array}$ & $1.49_{0}$ & 3.64 & 0 & 1.0 & 0 & 0 \\
\hline$s \mathrm{MA}^{\mathrm{a}}$ & Benzoin-UV & Bulk at $-50^{\circ} \mathrm{C}$ & $0.62_{5}$ & 1.40 & 1.0 & 0.03 & 0.16 & 0.81 \\
\hline$s \mathrm{MA}-2$ & Benzoin-UV & Bulk at $-50^{\circ} \mathrm{C}$ & $0.98_{6}$ & 2.46 & 1.0 & 0.04 & 0.16 & 0.80 \\
\hline AMA & $\left(\mathrm{C}_{6} \mathrm{H}_{5}\right)_{2} \mathrm{Na}$ & $\begin{array}{l}\text { THF soln. }{ }^{b} \\
\text { at }-78^{\circ} \mathrm{C}\end{array}$ & $\ldots$ & $3.43^{\mathrm{c}}$ & 0.70 & 0.07 & 0.40 & 0.53 \\
\hline Dpm-M & $\left(\mathrm{C}_{6} \mathrm{H}_{5}\right)_{2} \mathrm{Mg}$ & $\begin{array}{l}\text { Toluene soln. } \\
\text { at }-50^{\circ} \mathrm{C}\end{array}$ & $0.84_{2}$ & 2.02 & 0.60 & 0.39 & 0.11 & 0.50 \\
\hline Bmc-M & $\mathrm{CH}_{3}\left(\mathrm{CH}_{2}\right)_{3} \mathrm{MgCl}$ & $\begin{array}{l}\text { Toluene soln. } \\
\text { at }-50^{\circ} \mathrm{C}\end{array}$ & $1.05_{0}$ & 2.66 & 0.57 & 0.32 & 0.11 & 0.57 \\
\hline $\begin{array}{ll}\text { a } & \text { Unfra } \\
\text { b } & \text { THF: } \\
\text { c } & \text { Numb }\end{array}$ & $\begin{array}{l}\text { ated samples. } \\
\text { ahydrofuran. }\end{array}$ & & & & & & & \\
\hline
\end{tabular}




\section{T. MiYAMOTO and H. INAGAKI}

were recovered and used for subsequent experiments, and were coded as Bmc-M and Dpm-M in accordance with the former and the latter catalyst employed.

\section{Characterization of Samples}

The characterization of these samples was made by viscometry, and by infrared and highresolution NMR spectroscopy. Intrinsic viscosities [ $\eta]$ were determined in chloroform at $25^{\circ} \mathrm{C}$ and average molecular weights $M_{v}$ were estimated by using a relation

$$
[\eta] 25^{\circ} \mathrm{C}=4.8 \times 10^{-5} M_{w}^{0.8},
$$

which has been established for conventional PMMA. ${ }^{16}$ The $2-15 \mu$ spectra were taken with a Perkin-Elmer Model 521 spectrophotometer. The infrared index $\mathrm{A}$, which is defined by the ratio of absorbances at 7.25 and $9.45 \mu$, was determined and used as a measure of the tacticity of samples. The $\mathrm{nmr}$ spectra were taken with a Varian A-60 spectrometer from $5-10 \%$ solutions in $\mathrm{CDCL}_{3}$ at room temperature. Using the NMR data, the tacticity was assessed in accordance with the method developed by Bovey and Tiers. ${ }^{17}$ Results of the characterization are summarized in Table I together with the polymerization conditions.

\section{Other Experimental Techniques}

The experimental procedure of TLC employed has been described in detail in our previous works which demonstrated its successful application to studies on the chemical structure of binary copolymers ${ }^{18,19}$ as well as on the steric isomerism of PMMA. ${ }^{2,21}$ Silica gel was used for the stationary phase. The sample polymers were dissolved in chloroform at the concentration of $0.40 \mathrm{~g} / \mathrm{d} l$, and each spot on the thin layer was formed by $10 \mu \mathrm{g}$ polymer from the stock solution. On the basis of our previous results, ${ }^{20}$ ethyl acetate and acetone were employed as developers because of their different development characteristics; the former solvent develops only the syndiotactic (and atactic) PMMA, retaining the isotactic PMMA on the starting line, whereas the latter develops both types of PMMA at almost the same rate. The chromatogram thus obtained was stained with a $1 \%$ methanol solution of iodine for the determination of $\boldsymbol{R}_{f}$-value (rate of flow). It was often necessary to collect polymer species in each spot on the chromatogram in order to assign the steric structure of the developed components. For this purpose a thin layer of $1 \mathrm{~mm}$ thickness was utilized. The chromatogram was first stained and the portion of the thin layer containing the spot was scraped off the glass plate and treated with suitable solvents to extract the adsorbed polymer. More detailed descriptions of the procedure will be given in each case.

To investigate the features of the stereocomplex formation, especially as a function of time, velocity ultracentrifugation experiments were conducted at $25^{\circ} \mathrm{C}$ by using a Hitachi Ultracentrifuge Model UCA-1A with schlieren optics. Depending on the character of the test solution, a rotor speed was chosen in the range 7060 to $55430 \mathrm{rpm}$. X-ray photographs were taken by a Shimadzu X-ray diffractometer with a flat-plate camera, and the usual filtered $\mathrm{CuK} \alpha$ radiation was used. Further details of techniques used will be given below.

\section{RESULTS AND DISCUSSION}

\section{Stereocomplex Formation in Dilute Solution}

A short summary of our previous and new findings obtained for the stereocomplex formation by applying the techniques of TLC, ultracentrifugation, and X-ray diffraction will be given below. Mixtures prepared by admixing chloroform solutions $(0.40 \mathrm{~g} / \mathrm{d} l)$ of the two stereoregular polymers, $i \mathrm{MA}-3$ and $s \mathrm{MA}-2$, in three different ratios of $2: 1,1: 1$, and $1: 2$ (iso/synd) were subjected to the tlc experiment with acetone as the developer, taking into consideration that acetone can develop both isotactic and syndiotactic PMMA to the proximity of the solvent front. The chromatogram thus obtained showed that the 1:1 mixture could not be separated into components at all, whereas the other mixtures yielded a lower and an upper spot, as illustrated in Figure 1. Assignment by NMR indicated that for the $2: 1$ and $1: 2$ (iso/synd) mixtures, the components contained in the lower spots were identical with the $1: 1$ mixture; those in the upper spots for the 2:1 and 1:2 mixtures were the isotactic and syndiotactic polymers, respectively. ${ }^{20,21}$ On the basis of this result it was first concluded that this type of aggregation phenomena could occur stoichiometrically at a unique stereoisomeric composition of $1: 1 .^{21}$ 


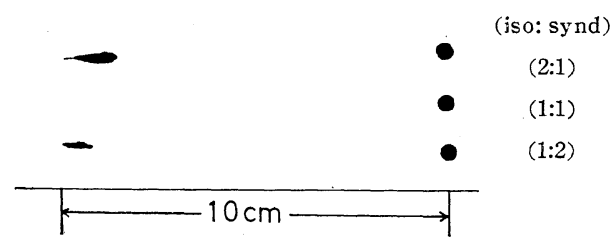

Figure 1. TLC chromatogram obtained for the mix tures of isotactic and syndiotactic PMMA in different mixing ratios using acetone as developer.

In addition it is of interest to note that to obtain the lower spot in such a distinct form as seen in Figure 1, the spot formed on the thin layer with the mixtures in chloroform had to be moistened with a drop of acetone and it was necessary to wait for a short time (e.g. 30-60 min.) before development. Nevertheless a slight ascendance of the lower spot after the development was unavoidable. This experience may be related to previous observations ${ }^{4}$ that the stereocomplex formation is accomplished more perfectly in polar solvents than in nonpolar solvents, and also implies that the stereocomplex formation is of a time-dependent nature.

On the other hand, results of some visible observations on the stereocomplex formation should be mentioned. The turbidity appearing upon admixture of dilute acetone solutions of isotactic and syndiotactic PMMA exhibits distinctly higher values in the $1: 2$ (iso/synd) ratio than in the $1: 1$ ratio, probably as the result of the stereocomplex formation, the interpretation for this observation being in agreement with that proposed by other authors. $^{2,4}$ For the 1:2 mixture a perfect phase separation can be visibly detected if the polymer concentration reaches $0.1 \%$. In contrast to this observation, such a phase separation does not take place for the 1:1 mixture even at $0.6 \%$ after the sample solutions are equilibrated for a few days, and appears first at a concentration above $0.9 \%$. This situation is similar to the $2: 1 \mathrm{mix}-$ ture, except that no phase separation occurs even above $1 \%$.

To relate the above observations to the stereocomplex formation, velocity ultracentrifugation experiments were carried out for the $1: 1$ and $2: 1$ (iso/synd) mixtures in acetone at a common concentration of $0.6 \%$. On the ultracentrifugation of the $1: 1$ mixture after the sample solution was equilibrated for 24 hours, a fast component appeared around $7000 \mathrm{rpm}$, showing a sharp peak in the schlieren pattern, while the slow component appeared first in a higher speed range beyond $51000 \mathrm{rpm}$ and showed flat peaks, as seen in Figures 2(a) and (b). The fast component may, of course, be identified with stereocomplexes of the $1: 1$ ratio; the slow component either free isotactic or syndiotactic PMMA. The reason for the existence of free PMMA molecules, though the number is very small, is still not clear; a possible interpretation is that the tacticity of the syndiotactic PMMA used here was lower than that of the isotactic polymer and/or that the polymer concentration was too low to form the stereocomplex in a $100 \%$ yield. In addition the difference in molecular weights of both samples might disturb the formation to some extent. Figures 3(a) and (b) illustrate the sedimentation patterns taken for the

(a)

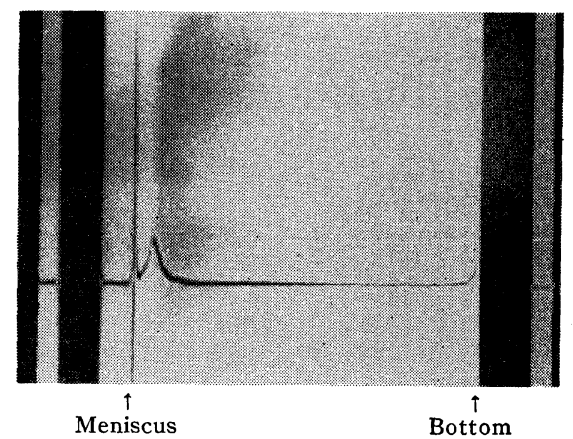

(b)

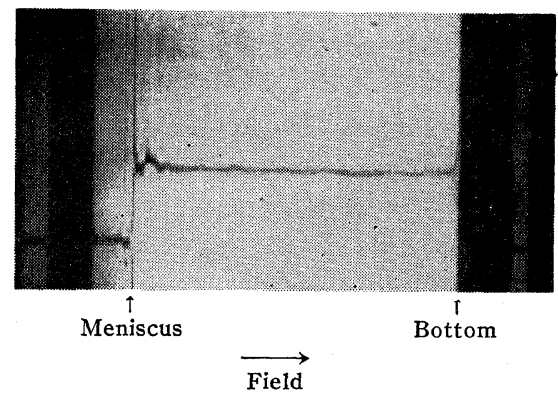

Figure 2. Sedimenta patterns obtained with $1: 1$ (iso/synd) mixture inacetone at $25^{\circ} \mathrm{C}$.

a: fast component: polymer concentration 0.6 $\mathrm{g} / \mathrm{d} l$; speed $7060 \mathrm{rpm}$; time $10 \mathrm{~min}$; phase plate angle $80^{\circ}$.

b: slow component: polymer concentration 0.6 $\mathrm{g} / \mathrm{d} l$; speed $51200 \mathrm{rpm}$; time $20 \mathrm{~min}$; phase plate angle $70^{\circ}$. 
(a)

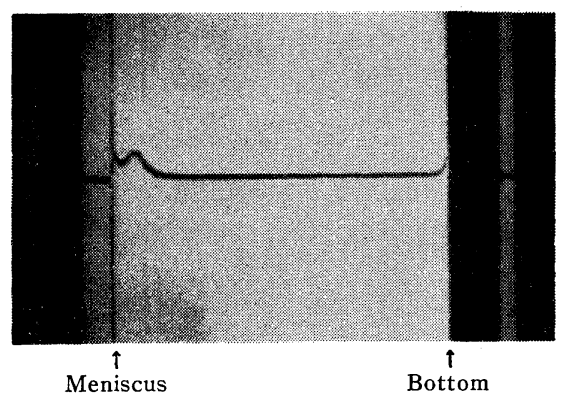

(b)

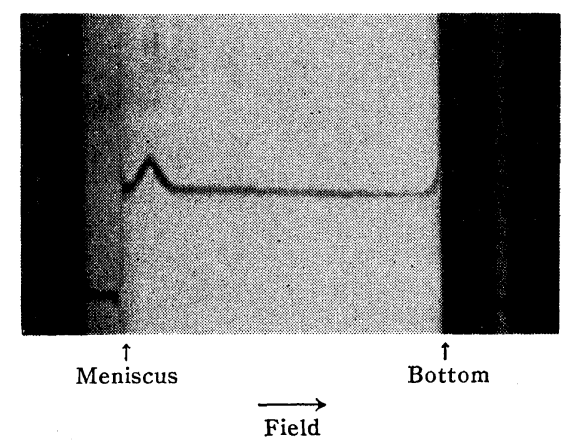

Figure 3. Sedimentation patterns obtained with $2: 1$ (iso/synd) mixture in acetone at $25^{\circ} \mathrm{C}$.

a: fast component: polymer concentration 0.6 $\mathrm{g} / \mathrm{d} l$; speed $14000 \mathrm{rpm}$; time $10 \mathrm{~min}$; phase plate angle $80^{\circ}$.

b: slow component: polymer concentration 0.6 $\mathrm{g} / \mathrm{d}$; s speed $55430 \mathrm{rpm}$; time $20 \mathrm{~min}$; phase plate angle $70^{\circ}$.

2:1 mixture at lower and higher rotor speeds (ca. 14000 and $55430 \mathrm{rpm}$.), respectively. This result clearly demonstrates that in contrast with the 1:1 mixture, a large number of the free PMMA molecules exist in the 2:1 mixture, although the stereocomplex (the fast component) is formed in the 2:1 mixture as well as in the 1:1 mixture. These ultracentrifugation results are consistent with the visible observations mentioned above.

Next we will describe the structural features of the stereocomplex obtained from the 1:2 (iso/ synd) mixture in acetone. As was stated, this mixture separates into gel and sol phases when the polymer concentration exceeds $0.1 \%$. The stereocomplex was recovered in powder form from the gel phase by filtration followed by drying in a vacuum at room temperature. An X-ray diffraction pattern was taken of this sample in powder form and is shown in Figure 4; it indicates that the sample has a high crystallinity though no fiber diagram was seen. We made no pretreatment, such as hot drawing of the sample, as other authors did, ${ }^{2 a}$ since we attempted to avoid any change in the crystallographic structure of the sample. In contrast to Figure 4, a film prepared with the $1: 2$ (iso/synd) mixture in chloroform had an extremely low crystallinity, as shown in Figure 5, even when the film was subjected to hot drawing at $60^{\circ} \mathrm{C}$. This result may support the conclusion drawn by $\mathrm{Liu}$ and $\mathrm{Liu}^{4}$ with $\mathrm{nmr}$ spectra, viz., that the formation of stereocomplexes is not accomplished by intermolecular entangle-

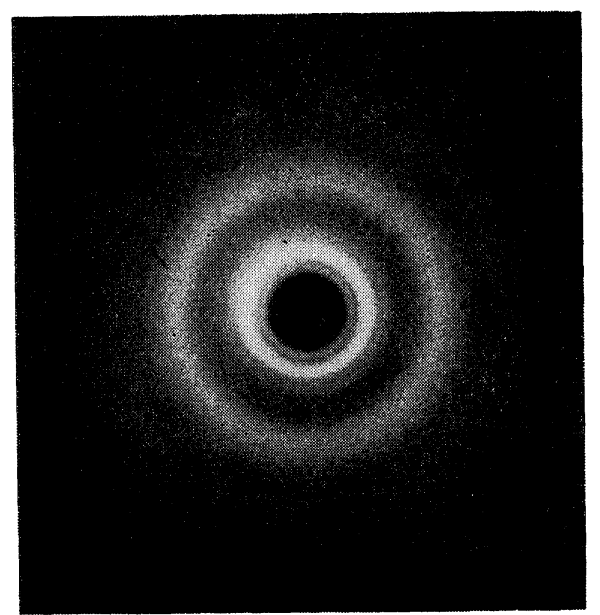

Figure 4. X-ray diffraction pattern of the stereocomplex obtained from the $1: 2$ (iso/synd) mixture in acetone.

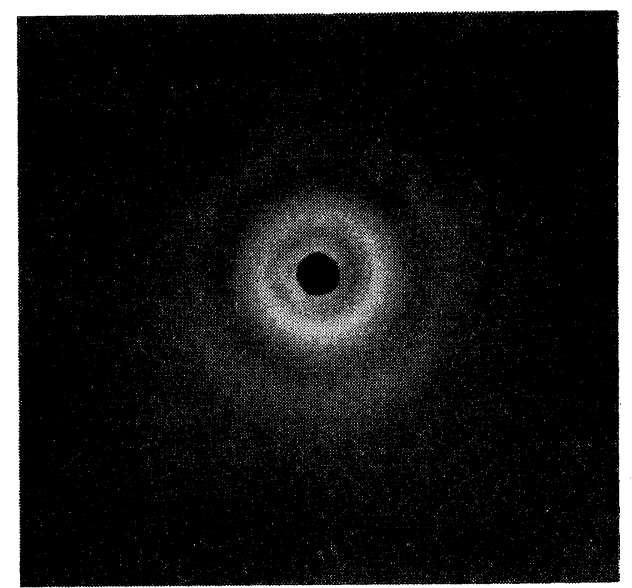

Figure 5. X-ray diffraction pattern of the film prepared with the 1:2 (iso/synd) mixture in chloroform. 
ments but rather by associations among considerably long sequences of different stereoisomeric chains.

On the basis of these experimental results we emphasize that the stereocomplex with the stereoisomeric composition of $1: 1$ will be formed as the primary structure. This conclusion appears somewhat inconsistent with those proposed by other authors $^{2-5}$ as well as our visible observations, but this inconsistency may be interpreted in terms of the mechanism of the stereocomplex formation. After the 1:1 stereocomplex formation has been accomplished, then associations between the stereocomplex and free syndiotactic molecules, if they exist in solution, occur to form the 1:2 (iso/synd) stereocomplex. Thus the formation of the 1:2 stereocomplex depends on whether the syndiotactic molecules are available in solution after the $1: 1$ stereocomplex has been formed.

In this connection it should be remembered that the 1:2 (iso/synd) mixture was separated into the component of the syndiotactic polymer and that of the $1: 1$ mixture. However, this should not be taken to mean that the $1: 1$ stereocomplex is the only possible association form. To clarify this result it should be understood that the tlc development generally depends on a competitive balance of interactions of three types, viz., polymersolvent, solvent-silica (stationary phase), and silicapolymer. ${ }^{18,19}$ Thus our TLC result could be rather intuitive evidence for the presumption that the $1: 2$ stereocomplex would be formed through looser interactions between the 1:1 stereocomplex and the syndiotactic molecules than between the isotactic and syndiotactic molecules. In other words, the 1:1 stereocomplex is the primary structure, while the 1:2 (iso/synd) stereocomplex is the secondary one.

\section{Steric Structure of So-called "Stereoblock" PMMA}

Using the same tlc procedure employed for the stereoisomeric mixtures, we investigated the development characteristics of the stereoblock samples Bmc-M and Dpm-M. For this purpose ethyl acetate was used as developer, because it can develop only the syndiotactic (and atactic) PMMA. Figure 6 shows the chromatogram thus obtained, in which the other two species, i.e. the 1:2 (iso/synd) mixture and an atactic PMMA (AMA), were developed simultaneously for com-

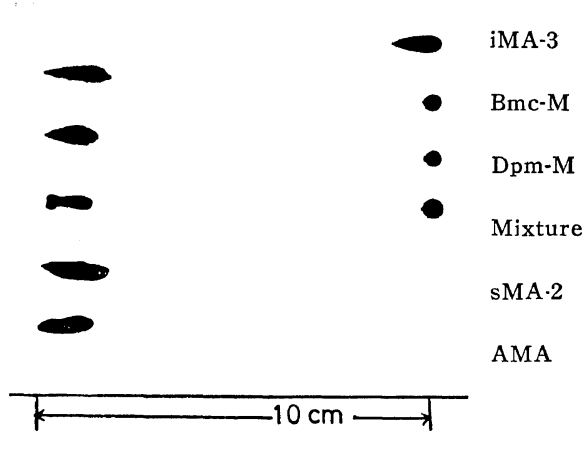

Figure 6. TLC chromatogram obtained by development of different stereoisomeric types of PMMA with ethyl acetate, viz. isotactic ( $i \mathrm{MA})$, stereoblock (Bmc-M and Dpm-M), the 1:2 mixture, syndiotactic (sMA-2), and atactic (AMA) PMMA, in order from top to bottom.

parison. The result indicates that the samples Bmc-M and Dpm-M are separated into an upper and a lower spot.

The polymer species contained in each spot were recovered, as described in the "Experimental Section", and subjected to NMR analysis. In connection with the extraction of the polymer from the thin layer it is worthwhile mentioning that both species thus separated by TLC were no more insoluble in acetone, although the sample had been originally obtained as the portion insoluble in hot acetone. From an inspection of the nmr spectra taken for the species contained in the upper and the lower spot we concluded that the main components involved in the upper and the lower spot were assigned to the syndiotactic and the isotactic PMMA, respectively, though these polymers contained some amounts of the heterotactic sequences. ${ }^{21}$

The relative amount of the two components separated from sample Dpm-M was determined by a photographic procedure established previously. ${ }^{20}$ This method is based on the experimental finding that the blackness of spots on photofilms of chromatograms stained with methanol solution of iodine is proportional to the amount of PMMA contained within a limited range ( $>c a .180 \mu \mathrm{g} / \mathrm{cm}^{2}$ ) and the proportionality constant for the isotactic PMMA is larger by a 


\section{T. MiYamoto and H. InAGaki}

factor of $1.3_{2}$ than that for the syndiotactic PMMA. ${ }^{20}$ Using the photofilm of the chromatogram in this way, the area and blackness of the spot were measured. The total blackness corrected against the area was converted to the amount of polymer by taking the factor of $1.3_{2}$ into account. The final evaluation of the relative amount was 0.71:0.29 for the syndiotactic and the isotactic portion, respectively, and this is in general agreement with the result reported by Liquori and his associates for a similar type of PMMA prepared with 9-fluorenyllithium. ${ }^{12}$ Thus we may conclude that the $1: 2$ (iso/synd) stereocomplex is formed during stereospecific polymerization with different Grignard's types of catalysts.

Stereoregular Polymerizations in the Presence of Tactic Polymers

It should be pointed out that the polymerization mechanism proposed by Coleman and Fox for the stereoblock formation ${ }^{10,11}$ cannot be reconciled with the conclusion drawn from these experimental results. At the same time, the conclusion does allow us to assume that the essential step in polymerizations of this type may be conditioned by the stereocomplex formation. Such a consideration leads us to believe that if the polymerization is carried out in the presence of tactic PMMA, the addition of the polymer should affect the rate of polymerization, the steric character of resultant polymers, etc.

Two preliminary stereospecific polymerization runs of MMA were made at $-50^{\circ} \mathrm{C}$ with $n$-butylmagnesium chloride in toluene in the presence of stereoregular PMMA. $17 \mathrm{ml}$ of monomer (MMA) and either $1.6 \mathrm{~g}$ of isotactic PMMA or $2.0 \mathrm{~g}$ of syndiotactic PMMA were first dissolved in $200 \mathrm{ml}$ of toluene. To this mixture $0.012 \mathrm{~mol}$ of $n$ butylmagnesium chloride dissolved in diethyl ether $(1 \mathrm{ml})$ was added at $-50^{\circ} \mathrm{C}$ to initiate polymerization. In this manner two polymerization runs were performed in the presence of the isotactic polymer (I-series) and another was performed in the presence of the syndiotactic polymer (S-series). In addition three polymerization runs were conducted in the absence of the stereoregular polymer but under the same conditions as the above (B-series). Here it must be mentioned that the polymerization proceeded with an accompanying formation of gel phase, which appeared to be due to the stereocomplex formation. In each polymerization, the reaction was terminated by pouring it rapidly into an excess amount of methanol. The whole polymers which were composed of the added and the produced polymer were purified by twofold precipitation from chloroform into methanol, followed by vacuum drying at $60^{\circ} \mathrm{C}$. Table II summarizes the polymerization data. The results show that not only the addition of stereoregular PMMA, but also the difference in the steric isomerism of added PMMA, distinctly affect the rate of polymerization. The rate of polymerization in the I-series appeared to be faster than that in the B-series, whereas the presence of syndiotactic polymer in the S-series had little effect on it.

As to the whole polymers thus obtained, it would be expected that if the produced polymer were stereoregular, the whole polymer would contain the stereocomplex, which is insoluble in boiling acetone. ${ }^{21}$ Therefore each whole polymer was subjected to extraction with acetone at boiling point. In fact the whole polymers were separated

Table II. Polymerization data of MMA with $n$-butylmagnesium chloride in toluene at $-50^{\circ} \mathrm{C}$

\begin{tabular}{cccccc}
\hline $\begin{array}{c}\text { Polymer } \\
\text { code }\end{array}$ & $\begin{array}{c}\text { Initiator } \\
(\mathrm{mol} / l)\end{array}$ & $\begin{array}{c}\text { Monomer } \\
(\mathrm{mol} / l)\end{array}$ & $\begin{array}{c}\text { Added polymer } \\
(\mathrm{g} / l)\end{array}$ & $\begin{array}{c}\text { Time } \\
(\mathrm{min})\end{array}$ & $\begin{array}{c}\text { Conversion } \\
(\mathrm{wt} \%)\end{array}$ \\
\hline IRA-1 & 0.06 & 0.8 & 8 (iso.) & 15 & 9.5 \\
IRA-2 & 0.06 & 0.8 & 8 (iso.) & 30 & 33.0 \\
SRA-1 & 0.06 & 0.8 & 10 (synd.) & 15 & 17.0 \\
SRA-2 & 0.06 & 0.8 & 10 (synd.) & 30 & 23.4 \\
SRA-3 & 0.06 & 0.8 & 10 (synd.) & 60 & 33.0 \\
BMC-1 & 0.06 & 0.8 & 0 & 30 & 20.7 \\
BMC-2 & 0.06 & 0.8 & 0 & 60 & 27.1 \\
BMC-3 & 0.04 & 1.0 & 0 & 240 & 30.0 \\
\hline
\end{tabular}


into soluble and insoluble fractions, which will be henceforth designated by attaching. (s) and (i) respectively to the code of each starting fraction. The results are shown in Table III. As seen from the table, the addition of isotactic polymer decreased the acetone-soluble portion, while the products obtained in the S-series as well as in the B-series were rich in the acetone-soluble portion. Results of the characterization of these fractions and the tacticity of produced polymers are shown in Tables IV and V.

To ascertain whether the added polymer remained unchanged in its chain structure during
Table III. Results of acetone extract

\begin{tabular}{lccc}
\hline $\begin{array}{c}\text { Polymer } \\
\text { code }\end{array}$ & $\begin{array}{c}\text { Produced polymer } \\
\text { Added polymer }\end{array}$ & $\begin{array}{c}\text { Acetone extract } \\
\text { soluble } \\
(\%)\end{array}$ & $\begin{array}{c}\text { insoluble } \\
(\%)\end{array}$ \\
\hline IRA-1 & $1.1 / 1$ & 19 & 81 \\
IRA-2 & $3.6 / 1$ & 16 & 84 \\
SRA-1 & $1.6 / 1$ & - & - \\
SRA-2 & $2.2 / 1$ & 37 & 63 \\
SRA-3 & $3.1 / 1$ & 36 & 64 \\
BMC-1 & $\mathrm{a}$ & 31 & 69 \\
BMC-2 & $\mathrm{a}$ & 31 & 69 \\
BMC-3 & $\mathrm{a}$ & 32 & 68 \\
\hline
\end{tabular}

a See Table I for each conversion.

Table IV. Characterization of fractions

\begin{tabular}{|c|c|c|c|c|c|c|c|c|c|}
\hline \multirow{2}{*}{$\begin{array}{l}\text { Polymer } \\
\text { code }\end{array}$} & \multicolumn{3}{|c|}{ Tacticity } & \multirow{2}{*}{$\mathrm{I} /(\mathrm{H}+\mathrm{S})^{\mathrm{a}}$} & \multirow{2}{*}{$\begin{array}{l}{[\eta](\mathrm{ml} / \mathrm{g})} \\
\text { chloroform } \\
25^{\circ} \mathrm{C}\end{array}$} & \multirow{2}{*}{$\mathbf{M}_{v} \times 10^{-4}$} & \multicolumn{3}{|c|}{ GPC data } \\
\hline & I & $\mathrm{H}$ & $\mathrm{S}$ & & & & $M_{w} \times 10^{-4}$ & $M_{n} \times 10^{-4}$ & $M_{w} / M_{n}$ \\
\hline IRA-1 & 0.55 & 0.15 & 0.30 & & & & & & \\
\hline IRA-2 & 0.52 & 0.15 & 0.33 & & & & & & \\
\hline IRA-2(i) & 0.50 & 0.13 & 0.37 & $1 / 1$ & 1.310 & 35.1 & & & \\
\hline IRA-2(s) & & & & & $0.17_{7}$ & 2.88 & 5.15 & 3.07 & 1.68 \\
\hline SRA-2 & 0.30 & 0.23 & 0.47 & & & & & & \\
\hline SRA-3 & 0.30 & 0.18 & 0.52 & & & & & & \\
\hline SRA-3(i) & 0.33 & 0.12 & 0.55 & $1 / 2$ & $0.73_{0}$ & 22.5 & & & \\
\hline SRA-3(s) & & & & & $0.23_{3}$ & 4.06 & 8.60 & 3.41 & 2.52 \\
\hline BMC-3(i) & 0.32 & 0.11 & 0.57 & $1 / 2$ & 1.050 & 26.6 & & & \\
\hline $\mathrm{BMC}-3(\mathrm{~s})$ & 0.26 & 0.34 & 0.40 & & $0.46_{8}$ & 9.68 & 20.2 & 4.48 & 4.51 \\
\hline
\end{tabular}

a $\mathrm{I} /(\mathrm{H}+\mathrm{S})$ : the isotactic/(heterotactic + syndiotactic) structure ratio in the triad.

Table V. Tacticity of produced polymers

\begin{tabular}{cccc}
\hline \multirow{2}{*}{$\begin{array}{c}\text { Polymer } \\
\text { code }\end{array}$} & \multicolumn{3}{c}{ Tacticity } \\
\cline { 2 - 4 } & I & H & S \\
\hline IRA -1 & 0.13 & 0.29 & 0.58 \\
SRA -2 & 0.34 & 0.24 & 0.42 \\
BMC-3 & 0.30 & 0.18 & 0.52 \\
\hline
\end{tabular}

polymerization, the tlc technique was used. Samples IRA-2(i) and SRA-3(i) were developed with ethyl acetate. The chromatograms thus obtained indicated that both samples were distinctly separated into an upper and a lower spot, which were assigned to the syndiotactic and the isotactic PMMA, respectively. ${ }^{20}$ It was also proved by intrinsic viscosity measurements that no changes in the molecular weight of added polymers had occurred.

Inspection of the results described above suggests that not only the addition of stereoregular
PMMA, but also the difference in the steric isomerism of added PMMA, distinctly affect the steric structure of produced polymers. The configuration of produced polymers obtained in the I-series was predominantly syndiotactic in low conversion, whereas those in the S-series contained isotactic and syndiotactic sequences as well. The isotactic/(heterotactic + syndiotactic) structure ratios in the triad, $I /(H+S)$ of the acetone-insoluble portions from the $\mathrm{S}$ - and I-series were $1: 2$ and $1: 1$, respectively, so far as the conversions of monomer to polymer are as low as those studied here. The gel permeation chromatography (GPC) studies show that the acetonesoluble portions derived from the I-Series are low in molecular weight and narrow in molecular weight distribution. These findings indicate that the existence of isotactic polymer chains promotes preferentially the polymerization of MMA to syndiotactic polymer chains, the reaction being 


\section{T. MIYAMoto and H. INAGAKI}

likely to proceed as a replica polymerization. On the other hand, the existence of syndiotactic polymer chains seems to be a prerequisite for initiating the polymerization of MMA to isotactic polymer chains so far as this catalytic system is employed at $-50^{\circ} \mathrm{C}$. In this connection it should be recalled that this catalytic system results in the stereocomplex of $1: 2$ (iso/synd) ratio even without adding syndiotactic PMMA to the system.

A small amount of diethyl ether, in which the Grignard reagent is prepared, will influence the configuration of produced polymers. ${ }^{9}$ However, it is probable that such an effect is negligible in the present case, because the presence of diethyl ether will have the same relative effect on the steric structure of produced polymers.

In conclusion we wish to propose a specific polymerization mechanism that a distinct array of MMA molecules along the polymer chain is energetically profitable with the result that a stereospecific incorporation of the monomer into a distinct pattern occurs, as suggested first by Szwarc. $^{22}$ This stereospecific polymerization mechanism might be closely connected with the stereocomplex formation. ${ }^{1-6,21}$

These observed effects could be influenced by the molecular weight of added polymers. Unfortunately, the molecular weights of added isotactic and syndiotactic polymers used in this study are quite different. A more detailed investigation of this polymerization mechanism is in progress in our laboratory, especially in relation to the effects of molecular weight and amount of added tactic PMMA on the stereoisomeric composition of acetone-insoluble portions.

Acknowledgement. We wish to thank Mr. N. Donkai for his technical assistance in the ultracentrifuge experiments.

\section{REFERENCES}

1. (a) W. H. Watanabe, C. F. Ryan, P. C. Fleisher, Jr., and B. S. Garrett, J. Phys. Chem., 65, 896 (1961); (b) C. F. Ryan and P. C. Fleisher, Jr., ibid., 69, 3384 (1965).
2. (a) A. M. Liquori, G. Anzuino, M. D'Alagni, P. De Santis, and M. Savino, Nature, 206, 358 (1965); (b) A. M. Liquori, M. De Santis, M. Savino, and M. D'Alagni, J. Polym. Sci., Part B, 4, 943 (1966).

3. R. Chiang, J. J. Burke, J. O. Threlkeld, and T. A. Orofino, J. Phys. Chem., 70, 3591 (1966).

4. H. Z. Liu and Kan-Jen Liu, Macromolecules, 1, 157 (1968).

5. J. Dayantis, C. Reiss, and H. Benoit, Makromol. Chem., 120, 113 (1968).

6. W. B. Van Den Berg, B. Hijimans, P. Piet, and D. Heikens, Nature, 217, 949 (1968).

7. T. G. Fox, B. S. Garrett, W. E. Goode, S. Gratch, J. F. Kincaid, A. Spell, and J. D. Stroupe, $J$. Amer. Chem. Soc., 80, 1768 (1958).

8. J. D. Stroupe and R. E. Hughes, J. Amer. Chem. Soc., 80, 2341 (1958).

9. W. E. Goode, F. H. Owens, R. P. Fellmann, W. H. Snyder, and J. E. Moore, J. Polym. Sci., 46, 317 (1960).

10. B. D. Coleman and T. G. Fox, J. Chem. Phys., 38, 1065 (1963).

11. B. D. Coleman and T. G. Fox, J. Polym. Scl. Part C, 4, 345 (1964).

12. A. M. Liquori, G. Anzuino, M. D'Alagni, V. Vitagliano, and L. Constantino, J. Polym. Sci., Part A-2, 6, 509 (1968).

13. V. Crescenzi, M. D'Alagni, A. M. Liquori, L. Picozzi, and M. Savino, Ric. Sci., 33, 123 (1963).

14. T. G. Fox, W. E. Goode, S. Gratch, C. M. Huggett, J. F. Kincaid, A. Spell, and J. D. Stroupe, J. Polym. Sci., 31, 173 (1958).

15. A. Roig, J. E. Fiqueruelo, and E. Llano, J. Polym. Sci., Part B, 3, 171 (1965).

16. J. Bisschoff and V. Desreux, Bull. Soc. Chim. Belges., 61, 10 (1952).

17. F. A. Bovey and G. V. D. Tiers, J. Polym. Sci., 44, 173 (1960).

18. H. Inagaki, H. Matsuda, and F. Kamiyama, Macromolecules, 1, 520 (1968).

19. F. Kamiyama, H. Matsuda, and H. Inagaki, Makromol. Chem., 125, 286 (1969).

20. H. Inagaki, T. Miyamoto, and F. Kamiyama, J. Polym. Sci., Part B, 7, 329 (1969).

21. T. Miyamoto and H. Inagaki, Macromolecules, 2, 554 (1969).

22. M. Szwarc, J. Polym. Sci., 13, 317 (1954). 